Théologiques

Théologiques

\title{
Autour de la question de Dieu : l'interdisciplinarité!?
}

\section{Gilbert Renaud}

Volume 6, numéro 2, octobre 1998

Dieu interdit

URI : https://id.erudit.org/iderudit/024959ar

DOI : https://doi.org/10.7202/024959ar

Aller au sommaire du numéro

\section{Éditeur(s)}

Faculté de théologie de l'Université de Montréal

\section{ISSN}

1188-7109 (imprimé)

1492-1413 (numérique)

Découvrir la revue

\section{Citer cet article}

Renaud, G. (1998). Autour de la question de Dieu : l'interdisciplinarité!?

Théologiques, 6(2), 7-24. https://doi.org/10.7202/024959ar

\section{Résumé de l'article}

Posant d'abord la question de la construction de l'interdisciplinarité entre les sciences humaines et la théologie, cet article tente de faire le point sur la question de Dieu dont l'interdit généré par les sciences humaines semble avoir reflué sur la théologie. Il examine ensuite les malaises produits par un social que les sciences humaines ont fortement contribué à produire en le vidant de toute question transcendantale et il ébauche finalement un certain nombre de questions qui pourraient servir d'amorce à une possible interdisciplinarité. 
Théologiques 6/2 (1998) 7-24.

\section{Autour de la question de Dieu : I'interdisciplinarité!?}

Gilbert RENAUD

École de service social Université de Montréal

De manière peut-être un peu abrupte, j'aimerais d'abord préciser qu'il me semble étrange de retrouver en milieu universitaire une faculté de théologie. Mon penchant laïque et mon appartenance à l'univers des sciences humaines me rendent spontanément incompréhensible le maintien d'une telle faculté au sein de l'université et je serais plutôt tenté de n'y voir que les vestiges d'une certaine tradition qui tarde à mourir. J'éprouve certes un grand respect pour ceux et celles qui y œuvrent et je suis fasciné par leur capacité d'assumer ce qui me semble être une position contradictoire : professer la théolo gie tout en participant à l'univers moderne et scientifique de l'université. En fait, ils ne me semblent parvenir à résoudre cette contradiction qu'en s'appuyant de plus en plus sur les sciences humaines auxquelles ils réfèrent abondamment pour légitimer le maintien d'un certain discours théologique. J'ai par conséquent l'impression que Dieu disparaît de l'univers de la théologie et qu'il faudra bien un jour que la faculté disparaisse elle-même à son tour pour faire place à un département de sciences religieuses, tandis que la pastorale pourra de son côté joindre les rangs du travail social dont elle ne se différencie souvent guère. En effet, il me semble toujours curieux de constater au fil d'échanges avec des collègues de pastorale que rien ne nous différencie vraiment quant à la question de l'intervention. Appartenant au monde du travail social, il m'arrive ainsi souvent de me demander ce que vient faire Dieu au sein d'un univers pastoral qui épouse les même visées psycho-sociales que le travail social?

Malgré toutes ces réserves, j'ai pourtant accepté de devenir membre du comité de rédaction de Théologiques. Il convient donc de 
préciser, en fonction de ce qui vient d'être énoncé, les raisons qui ont pu amener un professeur de l'École de service social, diplômé en service social et en sociologie, à se retrouver au comité de rédaction de la revue de la Faculté de théologie? Pourquoi, en outre, s'être montré intéressé par la co-direction d'un numéro qui aborde la question de Dieu à partir de l'interdit qui le caractérise au sein du discours universitaire, interdit que les sciences humaines ont précisément contribué à parachever?

Il est bien évident que dans le champ des sciences humaines, la question de Dieu prête généralement à sourire lorsqu'elle ne suscite pas un fort ressentiment anti-clérical. Il y belle lurette qu'on lui a réglé son compte et, tout au plus, peut-on accepter qu'il s'agit là d'une question strictement personnelle et privée, et que la théologie ne peut, par conséquent, en aucune façon contribuer au développement de la connaissance, à moins qu'elle ne devienne pleincment science religieuse...

Dans une telle perspective, l'interdisciplinarité est carrément impossible et impensable. En fait, elle ne peut s'exercer qu'à sens unique : des sciences humaines vers la théologie qui en viendra alors forcément à se convertir définitivement au credo scientifique et à la vision moderne du monde. Finie, une fois pour toutes, la question de Dieu : à la théologie pourra succéder une "religiologie" qui ne s'intéressera plus à Dieu et au phénomène religieux que sous l'angle historique, anthropologique et sociologique. Est-ce là le seul scénario possible? La question mérite qu'on s'y arrête, d'autant plus que Théologiques se définit comme "une revue de recherche interdisciplinaire, qui entend promouvoir le développement de la recherche en théologie, en dialogue avec les sciences humaines." Une réflexion s'impose donc dans la perspective de ce qui pourrait consti tuer une véritable interdisciplinarité, c'est-à-dire une inter-féconda tion réciproque de nos discours qui n'occulte pas, mais reconnaît et accepte plutôt le fondement différencié des sciences humaines et de la théologie. Et puisque le fondement de cette dernière me semble être la question de Dieu, question complètement absente de l'univers auquel j'appartiens, il importe de situer le cheminement/questionnement qui m'a conduit à accepter de relever le défi de participer au comité de rédaction de Théologiques. 
On l'aura compris, cet article épouse davantage les perspectives de l'essai et il n'a d'autre visée que d'amorcer un dialogue avec la théologie afin que puisse s'ébaucher cette interdisciplinarité souhaitée par Théologiques. Il reflète le questionnement d'un Québécois approchant la cinquantaine. C'est dire qu'il a vécu son enfance sous la tutelle de l'Église dont il a commencé à s'émanciper lors de la Révolution tranquille, tandis que sa formation universitaire en service social et en sociologie allait définitivement régler la question d'un Dieu devenu parfaitement inutile pour la compréhension et le fonctionnement du monde. Autrement dit, ce cheminement reflète une banale histoire de modernisation personnalisée qui s'est construite comme processus d'émancipation à l'intérieur duquel Dieu a été toujours davantage interdit et mis à mort!

\section{Le service social et la question de Dieu}

Il est bien évident que, tel qu'il se présente aujourd'hui, le service social québécois s'est largement émancipé de toute référence à Dieu. L'idée de Dieu y apparaît complètement incongrue et impertinente ("irrelevant ") en regard de la gestion des problèmes sociaux auxquels les praticiens sont confrontés. Pourtant, il n'est pas encore si loin le temps où le discours légitimant puisait largement dans la doctrine sociale de l'Église. À cet égard, deux publications datant des origines de la formation universitaire en service social ne manquent pas d'être fort évocatrices : ainsi, le père Émile Bouvier, s.j., publiait en 1940 "Le samaritanisme moderne ou Service social ${ }^{1}$ "; tandis que quelques années plus tard, le père Georges-Henri Lévesque, o.p., y allait d'un "Service social et Charité ${ }^{2}$ ". Dieu merci, ces temps sont bien révolus!

À l'instar du Québec, le service social amorce son émancipation de la tutelle cléricale à partir des années 50 , tandis que les années 60 sonneront définitivement le glas de toute référence au discours religieux. En se libérant ainsi de l'emprise cléricale, le service social est entré résolument dans la voie de sa «scientifisation» au niveau universitaire et de sa rationalisation technocratique au plan organisa-

$1 \quad$ Publications de l'École sociale populaire, no 317, juin 1940.

2 Cahiers de la Faculté des Sciences sociales de l'Université Laval, Vol. III, no 2, 1944. 
tionnel. En définitive, il est passé du statut de charité appliquée (dont il ne faudrait pas oublier qu'elle a donné lieu à un système qui a trop souvent fait injure à la dignité humaine) à celui de science humaine appliquée... Si bien qu'au tournant des années 80 , les départements de service social se sont fortement engagés dans le sys tème de la recherche sociale et dans la course aux subventions qui le caractérisent. En fait, l'interconnexion entre la recherche et la gestion sociale y apparaît toujours plus étroite et l'on peut déjà à juste titre se demander ce qu'il advient alors de l'autonomie au fondement de la vie universitaire. Toujours mieux rodé, un véritable système d'ingénierie sociale se met en place où la recherche universitaire joue un rôle fondamental, ce que fait d'ailleurs bien ressortir un communiqué émis par le cabinet du Ministre de la santé et des services sociaux lors de la nomination du président du Conseil québécois de la recherche sociale en juin 1997 :

La recherche sociale est devenue, avec les années, une activité essentielle au fonctionnement du système de santé et de services sociaux. Elle est nécessaire tout autant à la planification qu'à l'intervention, tout autant à la décision politique qu'à la gestion. La recherche sociale permet, en effet, de connaître l'ampleur, la direction et les causes des problèmes sociaux afin de les prévenir ou de les régler. La recherche sociale répond aux questions de l'intervenant, oblige à l'expérimentation de nouvelles pratiques sociales et évalue l'efficacité ainsi que l'efficience des services et des programmes. Dans le contexte de la transformation du réseau de la santé et des services sociaux, la recherche joue un rôle crucial.

On ne saurait mieux exprimer l'étroite association entre la recherche sociale universitaire et la gestion des sociétés contemporaines. Tout irait pour le mieux dans le meilleur des mondes, si un tel accomplissement ne s'accompagnait du déclin de toute référence à une pensée synthétique globale et de la prééminence très grande de la pensée positiviste qui tend à faire du social un ensemble de problèmes sociaux qu'il faut appréhender de manière objective en les déployant comme autant d'indicateurs sociaux et de facteurs de risques en fonction desquels sont élaborés les programmes d'intervention. 
En fait, depuis le début des années 60, le développement du social dépasse largement le seul champ du service social. C'est bien davantage à la ramification du travail social (que l'on entendra au sens large en y agglomérant tous les métiers du social) à laquelle on a assisté. Il est remarquable en effet de constater l'extension considérable des champs de pratique spécialisée fondée sur un savoir instrumental qui s'est accomplie au fil de la modernisation de la société québécoise. Le social a sans cesse pris forme en s'immisçant dans les moindres replis de l'existence pour s'autoproduire comme système de gestion opérationnelle et efficace des populations qui lui sont soumises. En une formulation concise, Jean Baudrillard a su rendre compte du paradoxe auquel nous renvoie ainsi le social :

Si le social est fait des instances abstraites qui s'édifient les unes après les autres sur les ruines de l'édifice symbolique et rituel des sociétés antérieures, alors ces institutions en produisent de plus en plus. Mais en même temps elles consacrent cette abstraction dévorante, dévoratrice peut-être justement de la «substantifique moelle » du social. De ce point de vue, on peut dire que le social régresse à mesure même du développement de ses institutions ${ }^{3}$.

Le développement du social (et de la recherche qui le fonde) est ainsi allé de pair avec la multiplication de savoirs pratiques, instrumentaux et spécialisés qui s'étendent de la gérontologie à la récréologie (il existe même une récréo-gérontologie) en passant par la thanatologie, la sexologie, la toxicologie, en bref donc un ensemble de champs de pratique spécialisée issu du découpage incessant de l'existence, le tout pouvant être unifié sous le vocable de sciences humaines appliquées. Ce qui en résulte, c'est la production d'un système d'action guidé par des impératifs pragmatiques d'efficacité.

Ce mouvement, bien entendu, s'inscrit dans la primauté de la rationalité instrumentale qui définit à l'heure actuelle les sociétés contemporaines et assure à la pensée positiviste les assises de son développement. Plus globalement, ce mouvement participe luimême du processus de technocratisation qui travaille à la dissolution de l'idée classique de société (i.e. d'une totalité idéelle transcendantale conférant sens et signification à l'agir) et à la réduction de social. Paris, Denoël/Gonthier, 1982, p. 69-70. 
celle-ci à un réseau de systèmes opérationnels orientés de manière pragmatique vers l'efficacité (le règne du marché en étant la manifestation la plus explicite). La dynamique sociétale contemporaine nous renvoie ainsi de plus en plus à la dissolution de l'idée de société (qui a germé avec la modernité) et à l'organisation d'un système social qui engloutit toute finalité transcendantale au profit de l'instrumentalisation de l'existence personnelle et sociale. Le social tend alors à se déployer comme système de gestion efficace et technique des problèmes d'intégration qui peuvent se présenter. Pour le dire comme Michel Freitag 4 , on assiste au «développement d'une société postmoderne à caractère technologique, technocratique et systémique dans laquelle le concept de norme ne réfère plus à des valeurs synthétiques, mais seulement à des procédures et des procédés immédiatement opérationnels ». Le social perd donc sa dimension sociétale idéelle pour s'appréhender comme système soumis aux impératifs de la rationalité instrumentale.

Le service social ne fait donc ainsi que s'inscrire dans un courant plus global de technocratisation (ou de postmodernisation, comme le dirait Michel Freitag) où l'orientation de la recherche concourt à construire un social opérationalisé, fonctionnalisé et technicisé qui se fonde sur une représentation réduisant l'être humain à une mécanique vide produite par des rapports qui s'échelonnent sur un spectre allant du bio-chimique au psycho-social. Il ne peut en résulter que le développement de disciplines pratiques dont la présence et le poids se font sentir toujours davantage à l'université à travers la multiplication de diplômes et de certificats spécialisés; tandis que, de leur côté, les disciplines "traditionnelles" sont invitées à s'adapter à la réalité sociale contemporaine en faisant la preuve de leur utilité et de leur contribution à la gestion du système social (la pertinence sociale et les retombées prévisibles de la recherche!). C'est ainsi que la recherche joue son rôle crucial dans la construction du système, mais on en conclura peut-être comme Michel Freitag que cet apport s'éloigne de la compréhension de la réalité au profit de sa construction technocratique :

4 Michel FREITAG, Le naufrage de l'université. Et autres essais d'épistémologie politique. Québec, Nuit blanche Éditeur; Paris, La Découverte, 1995, p. 25. 
L'objectif des disciplines pratiques qui participent ainsi directement au fonctionnement de la réalité sociale contemporaine n'est donc plus tellement la connaissance ou la légitimation de cette réalité, mais directement sa production et son contrôle. La "théorie " tend donc à s'y confondre immédiatement avec la "pratique", au moment où cette pratique cesse de se penser comme praxis historique et qu'elle se banalise sous la forme d'une multitude d'activités toujours locales à caractère immédiatement pragmatique. La vérité tend alors à s'identifier à l'efficacité, la validité des problématiques, à la pertinence des stratégies de contrôle, et la méthode, à la fiabilité et à la prévisibi lité des procédés et des procédures techniques. À ce titre, il nous faut reconnaître d'ailleurs que les sciences sociales ainsi entendues sont devenues indispensables au fonctionnement des sociétés technocratiques contemporaines, et qu'elles appartiennent à leur concept même ${ }^{5}$.

En une cinquantaine d'années, on est donc ainsi passé de la charité chrétienne appliquée à la construction d'un social défini comme mode opérationnel de gestion des conduites et comportement humains. Alors, pour ce qui est de la question de Dieu, on comprend aisément qu'elle fasse figure d'archaïsme. Dès lors que le réductionnisme propre à la rationalité instrumentale laisse miroiter toute son efficacité et sa productivité, les humains peuvent espérer atteindre enfin cette toute-puissance qu'ils ont jadis projetée en Dieu...

\section{Malaise et interdit : vers l'interdisciplinarité?}

Telle est, me semble-t-il, la tendance lourde qui oriente princi palement le déploiement actuel du social et de la recherche qui sert à son édification. Et chacun aura compris que ma lecture relève d'un profond désenchantement à l'égard d'une telle perspective. Je me sens de fait bien étranger à l'euphorie scientiste qui gagne les milieux universitaires au fur et à mesure que s'accroît le montant des subventions obtenues. Je ressens plutôt un malaise profond devant cette entreprise où j'aperçois davantage une violence symbolique qui m'agresse, un vide de pensée qui me heurte... J'éprouve une crainte 
devant la perte qui nous guette, en même temps que je me demande s'il ne s'agit pas là tout simplement de la résistance de quelqu'un qui refuse le passage de la modernité à la postmodernité? Ne convient-il pas d'y voir en fait les récriminations d'un mésadapté enfermé dans une nostalgie passéiste d'un temps révolu, ce que confirmerait d'ailleurs sa présence à Théologiques? Faut-il arrêter ce qui apparaît souvent comme la déploration de ceux qui empêchent de tourner en rond pour plonger dans ce système où le moyen a supplanté la fin, ou, dit d'une autre manière, dans un système dont la visée principale n'est plus que sa propre effectuation?

Tout en restant vigilant par rapport à ces questions qui méritent d'être rappelées parce que l'idéalisation passéiste sert souvent de fondement à une lamentation sur les temps présents, il n'en reste pas moins que, pour ma part, je n'arrive tout simplement pas à croire que les humains ne sont rien d'autre que cette positivité à laquelle le scientisme les réduit avec les meilleures intentions du monde. Je n'arrive pas à acquiescer au fait que les conduites humaines ne relèveraient que d'une mécanique naturelle ou encore d'une physique sociale. Je persiste plutôt à penser que les humains se construisent à partir de la dimension symbolique qui les distingue de l'animalité. Autrement dit, je reste rivé à l'idée d'une humanité posée comme monde de l'esprit et je refuse sa réification et sa transformation en simple chose agie par des mécanismes biologiques, psychologiques et sociaux. Non pas parce qu'une telle manière de chercher à comprendre les comportements humains s'avère complètement fausse, mais plutôt parce qu'elle n'épuise pas la compréhension de la condition humaine et qu'elle ne saurait, en conséquence, en constituer le seul mode d'appréhension.

Découle alors de ces prémisses un questionnement relatif à cette humanité qui se constitue dans l'ordre symbolique au centre duquel apparaît la question du sens transcendantal qui caractérise l'agir humain. Que s'est-il donc passé? En ce qui concerne les sociétés modernes, Michel Freitag a bien montré qu'elles ne se constituent pas en abandonnant toute référence transcendantale, mais en déplaçant le point d'ancrage de cette référence. À la transcendance divine des sociétés pré-modernes succède ainsi une transcendance immanente: l'humanisme qui constitue en quelque sorte l'horizon transcendantal à partir duquel vont s'édifier les sociétés modernes. Mais ces sociétés semblent repousser toujours davantage la 
transcendance au profit de la seule immanence. C'est ainsi que l'on aboutit à la postmodernité où les sociétés cessent de se penser en fonction d'une quelconque référence transcendantale pour se concevoir comme système empirique, pragmatique, fonctionnel et opérationnel qui assure aux sciences de la gestion - sur lesquelles se calquent de plus en plus les sciences humaines - leur emprise toujours plus grande sur les socié tés contemporaines.

C'est bien ce déplacement qui confère au social sa forme actuelle et assure la ruine "de l'édifice symbolique et rituel des socié tés antérieures", pour reprendre les mots de Baudrillard. En fait, la société contemporaine s'érode comme système symbolique, c'està-dire comme instance pourvoyeuse de sens face aux événements dont est parsemée l'existence. Le social supplante la culture et il tend de plus en plus à n'offrir aux individus qu'un ensemble de procédures techniques qui indiquent les conduites à tenir mais qui laissent en suspens la question du sens. On comprend alors que le thème du vide social et de la perte de sens ait trouvé un si fort écho dans les sociétés contemporaines. Il correspond en fait à l'expérience d'individus de plus en plus laissés à eux-mêmes avec comme seul guide un impératif d'auto-réalisation de soi et de recherche de sens personnalisé. Le processus d'individualisation mis en marche par la modernité culmine ainsi aujourd'hui dans l'érosion de tout sens collectif invitant chacun à bricoler du sens, ainsi que le faisait ressortir David Le Breton:

L'individu tend de plus en plus à s'autoréférencer, à chercher dans ses ressources propres ce qu'il trouvait auparavant dans le système social de sens et de valeurs où s'inscrivait son existence. L'indétermination de nos sociétés, toujours en mouvement, le déracinement des repères collectifs projettent chaque individu vers une quête de sens fortement individualisée. Et chaque acteur ne peut aujourd'hui répondre que de façon personnelle à la question de la signification et de la valeur de son existence ${ }^{6}$.

Ainsi s'achève l'individualisation des sociétés qui atteint de tels sommets que l'on en est venu à parler d'un nouvel individualisme, ou encore d'un individualisme narcissique faisant ainsi référence à la progression de ce nouveau type de personnalité dont David Riesman 
avait déjà décelé, dans les années 50 , l'émergence dans la société américaine? $e^{7}$ Travail en quelque sorte de désocialisation où l'émancipation s'achève dans le déclin d'une culture collective capable de pourvoir chacun des repères lui permettant de construire son identité. Le social s'épanouit alors comme système techno-scientifique où la question du sens est délégitimée ne relevant plus que de la sphère personnelle. On comprend ainsi le climat de relativisation dans lequel baignent les sociétés et, comme le soulignait Ellen Corin, cette relativisation s'enracinerait encore plus fondamentalement dans une crise de la signifiance :

La postmodernité se caractérise donc moins par une crise des signifiants, qui inviterait à formuler de nouvelles valeurs, que par une crise de la signifiance, c'est-à-dire de la croyance en l'existence d'un centre au moins virtuel ou en la disponibilité de référents permettant de fonder un monde crédible, de décider du vrai et du faux, du bien et du mal, du plus et du moins ${ }^{8}$.

Dans cette perspective, tendent à ne plus subsister que les visées d'un système à même d'imposer sa logique et de délégitimer tout ce qui échappe à son propre développement. Alors, comment ne pas être tenté de conclure avec Luc Ferry que tout se passe "comme si devait disparaître, après la "mort de Dieu ", jusqu'au pressentiment d'une transcendance 9 ". Ou encore, comment ne pas acquiescer au constat de Yves Barel sur la difficulté des sociétés modernes à pro. duire du sens :

Les sociétés modernes, en particulier la société occidentale, ont un problème avec ce qui représente une des tâches fondamentales de toute société, la production de sens, et notamment de la

7 David RIESMAN, La foule solitaire. Anatomie de la société moderne. Paris, Arthaud, 1964.

8 Ellen CORIN, "Dérives des références et bricolages identitaires dans un contexte de postmodernité ", dans M. Elbaz et al. (dir.), Les frontières de l'identité. Modernité et postmodernité au Québec. Québec, Les Presses de l'Université Laval; Paris, L'Harmattan, 1996, p. 257.

9 Luc FERRY, L'homme-Dieu ou le Sens de la vie. Paris, Grasset, 1996, p. 96. 
forme la plus "noble" de ce dernier, qu'on désignera ici conventionnellement sous le nom de transcendance ${ }^{10}$.

On peut toujours arguer que toutes ces questions ne relèvent pas de la démarche scientifique, que la science doit se construire en dehors du sens et que les sciences humaines doivent, en conséquence, ignorer ce questionnement. Fort bien, mais en prétendant s'intéresser à la connaissance et à la compréhension des affaires humaines, on peut en retour se demander si elles auront encore quelque chose de fondamental à nous apprendre. De même, on peut toujours refuser de voir le problème en posant la question de la définition du sens, en soulignant que le sens persiste et en se réfugiant dans la poursuite d'un questionnement traditionnel qui, lui aussi, n'a plus bien souvent grand-chose à nous apprendre sur les temps présents. En fait, on peut changer de définition et même de vocabulaire, ne pas parler si l'on veut de sens ou de transcendance, mais il me semble difficile de ne pas reconnaître le nœud qui caractérise les sociétés contemporaines. Peu m'importent les termes, je reste d'abord préoccupé par une connaissance qui reconnaît les transformations en cours et les difficultés auxquelles elles nous renvoient. Autrement, il me semble qu'après la mort de Dieu, celle de "l'Homme " et du sujet, nous contribuons à la mort du sens et de la transcendance et que, même à notre corps défendant, nous soutenons les conquêtes positivistes qui en viennent, en matière d'intervention, par exemple, à réclamer la fin de l'enseignement de la psychanalyse, sous prétexte de sa nonscientificité ${ }^{11}$. Après Dieu et la théologie, Freud et la psychanalyse enfin rangés dans le rayon de l'histoire des idées pré-scientifiques: les humains pourront ainsi être plus facilement décortiqués telles des coquilles vides qu'ils seront effectivement devenus...

Mais, heureusement ou malheureusement - tout dépendant du point de vue -, ces humains semblent se rebiffer à la complète "désaliénation" que leur offre le monde contemporain. Le hic de toute cette histoire, c'est que subsistent des personnes et que celles-ci charrient avec elles des problèmes face auxquels le social semble

10 Yves BAREL, La quête du sens. Comment l'esprit vient a la cité. Paris, Seuil, 1987, p. 7.

11 Voir les propos tenus par Serge Larivée, professeur au département de psycho-éducation, dans Forum, vol. 31, no 2, 9 septembre 1996. 
souvent bien impuissant. C'est alors que l'on peut mesurer toute la distance qui sépare le monde de la gestion et celui des situations vécues. Comme l'a exprimé à sa manière Michel Freitag, les travailleurs du social subissent ainsi une double contrainte :

L'intention des "travailleurs sociaux" a sans doute (presque) toujours dépassé le concept de leur discipline, qui n'est rien d'autre que celui de la sociologie considérée comme un travail productif. Ce concept s'est alors servi d'eux, jusqu'au moment où il a fini, à leurs yeux mêmes, par se révéler plus fort que leurs intentions: alors, ce n'est pas le concept qui éclate, mais les intentions dont il fut chargé! Ce sont les idéaux normatifs qui chavirent dans le fonctionnement purement positif d'une société qui s'abandonne aux seules contraintes de son intégration systémique; ce sont les rêves politiques et éthiques qui se dissipent dans la grisaille cybernétisée de la gestion quotidienne du "social", cette nouvelle conception de la société dont les travailleurs sociaux n'auront hérité, en fin de compte, que le " mauvais côté ", celui où naissent sans cesse les "problèmes" parce qu'il y subsiste toujours encore des " personnes" 12 .

Et de ce mauvais côté de l'histoire, on remarque que «la société actuelle se caractérise ... par un accroissement sensible des pathologies relationnelles: dépression, "vide intérieur", angoisse, stress, anxiété, peur de l'avenir, sentiment d'inutilité...13" On pourrait aussi la caractériser à partir de l'augmentation des conduites extrêmes ${ }^{14}$, de la toxicomanie, des conduites de violence, de la délinquance, des problèmes de santé mentale, du burn out, des problèmes identitaires, du suicide (le décrochage ne semble pas le fait du seul appareil scolaire). À cela, on pourrait ajouter le développement des sectes, l'éclosion d'un marché noir de sang infecté par le virus du sida (dont on connaît l'existence sans être en mesure d'en évaluer l'étendue). En fait, la liste est longue de toutes ces conduites qui semblent renvoyer à une désorientation des individus, notamment parce que :

Michel FREITAG, op. cit., p. 186.

13 David LE BRETON, op. cit., p. 44.

14 Voir ibid. et Patrick BAUDRY, Le Corps Extrême. Approche sociologique des conduites à risque. Paris, L'Harmattan, 1991. 
Les principes rationnels ou formels qui guident aujourd'hui une large part des relations sociales sont dotés d'une moindre qualité anthropologique, ils laissent souvent démuni l'individu confronté à une épreuve personnelle (ou plutôt ce qui devient telle) : entrée dans la vie, avenir, maladie, accident, deuil, approche de la mort, solitude, vieillesse, handicap, chômage, etc. Ces événements inscrits dans la durée, en partie détachés des systèmes symboliques, sont vécus presque sans médiation, en une sorte de nudité. Leur résister en appelle à une créativité individuelle inégalement partagée, une capacité d'instaurer de nouveaux rituels, de se créer des valeurs personnelles, un réseau d'aide ou d'amitié. Si sa latitude d'action est élargie, l'individu est en revanche souvent désorientét ${ }^{15}$.

Faut-il alors que par rapport à chaque problème se développe un champ d'application? Faut-il, pour prendre cette image, continuer à s'intéresser à chaque arbre et en oublier la forêt? Faut-il poursuivre sur la même lancée en renforçant le développement d'une approche positiviste et technique des problèmes? En fait, les questions se bous culent qui me font douter de plus en plus de l'intérêt des sciences humaines appliquées et ce, d'autant plus que, ces dernières années, je me suis intéressé au domaine des soins palliatifs et que j'ai donc côtoyé la mort. J'y ai découvert bien souvent une spiritualité à l'eau de rose, une religiosité agaçante et des croyances ésotériques qui me font sourire, mais surtout j'y ai senti l'impuissance devant la finitude de la condition humaine, le vif questionnement qui taraude les intervenants et la recherche de sens qui y travaille chacun. J'ai pu alors bien mesurer la distance séparant le triomphalisme du travail social ou des sciences humaines appliquées et la détresse devant la mort, corrélative du déni dont elle est l'objet. Car après avoir mis à mort Dieu, "l'Homme", le sens, la transcendance, bref tout ce qui renvoie à l'ordre symbolique, les sociétés contemporaines aboutissent au déni de la mort qui ne manque pas de refluer sur nos conduites et nos rationalisations ${ }^{16}$ ! Curieuse trajectoire où désormais la mort est

David LE BRETON, op. cit., p. 43-44.

16 Pour prendre cet exemple révélateur, la quête d'excellence caractéristique des modes de gestion contemporaine refléterait ce déni de la mort (voir Thierry C. PAUCHANT, "La quête de l'excellence et le déni de la mort ", dans T. C. PAUCHANT et coll., La quête de sens. Gérer 
interdite au même titre que Dieu. Et pourtant, il en va de la mort comme de la vie...

C'est ainsi qu'à partir du malaise général (du mal-être ou du mal de vivre, peu importe la dénomination que l'on choisit) qui se manifeste de différentes manières surgit une méfiance de plus en plus accentuée à l'égard de sciences humaines axées sur leur opérationalisation. Et une question surgit alors : participant de plus en plus de la construction d'un social qui assure l'érosion du sens, de la transcendance et du symbolique, les sciences humaines ne concourent-elles pas à alimenter la production de problèmes qu'elles doivent gérer? Se peut-il en fait qu'en accentuant cette tendance, les sciences humaines pavent la voie de la désorientation d'individus à qui elles n'ont plus rien - ou de moins en moins - à offrir en regard de ce qui persiste malgré tout à transparaître? L'attrait pour l'irrationnel et la croissance des sectes ne sont-ils pas le reflet inversé du triomphe des sciences humaines qui ont fini à force de réductionnisme par vider les humains de leur humanité?

De ce point de vue, le tableau est, à vrai dire, déplorable mais il ne sert à rien, comme je l'ai déjà indiqué, d'entretenir une déploration des formes de vie sociale antérieure. Cela ne signifie évidemment pas qu'il faille à l'inverse acquiescer au réductionnisme dominant la pensée sociale. Apparaît plutôt un chantier qui requiert non pas de se réfugier dans le maintien des dogmes traditionnels (religieux ou scientifiques), mais qui appelle une sorte d'audace de la part de ceux qui, dans ce monde et son actualité, ne renoncent pas à faire œuvre de pensée. Il s'agit, somme toute, d'établir ce dialogue interdisciplinaire entre tous ceux qui aperçoivent dans l'humanité une sorte de mystère irréductible aux canons positivistes. À cet égard, la perspective interdisciplinaire avec la théologie peut peutêtre s'avérer intéressante et instructive, surtout si l'on est tenté de penser que nous avons peut-être encore besoin de la question de Dieu pour nourrir notre humanisation. Certes, il ne peut s'agir du côté des sciences humaines de se convertir à la croyance en un Dieu ainsi objectivé et réifié. Dieu restera toujours une création humaine mais

nos organisations pour la santé des personnes, de nos sociétés et de la nature. Montréal, Québec/Amérique, Presses HÉC, 1996, pp. 139-162). 
cela n'empêche pas d'essayer de comprendre ce qui vient à l'esprit humain à travers la question de Dieu. En sorte qu'à la transcendance, il faudra alors ajouter également le sacré, tant il appert que «... si les hommes n'étaient pas en quelque façon des dieux, ils ne seraient pas non plus des hommes. Il faut supposer en eux quelque chose de sacré ou bien accepter de les réduire à l'animalitél ${ }^{17}$. » On peut toujours décréter que ces questions ne sont pas pertinentes, qu'elles sont en fait irrecevables, étant donné qu'elles relèvent des illusions auxquelles les humains ont encore besoin de recourir dans leur aliénation et leur difficulté à assumer leur profond déterminisme. En fait, il n'est même guère possible d'en débattre, tant il est vrai que ces posi tions divergentes se fondent sur un ensemble de conceptions ou de représentations - de croyances - qui viennent orienter la réflexion. Les miennes me conduisent à penser - sans tomber dans une quelconque métaphysique du sujet - que la liberté agit aussi au cœur de la condition humaine.

\section{Amorce d'un dialogue avec la théologie}

C'est donc à partir de ce point de vue que peut s'ouvrir un dialogue avec la théologie. Mais avec quelle théologie ou avec quels théologiens? Bien sûr, d'abord avec ceux qui se sentent en affinité avec le portrait venant d'être présenté; mais cela ne saurait être suffisant dans la mesure où ceux-ci me semblent également aux prises avec l'interdit de Dieu qui les amène à se réfugier dans les sciences humaines, renforçant du coup ces mêmes sciences dans leur prétention à constituer le seul discours autorisé sur les "choses humaines". D'où me vient ce sentiment? Il convient de le préciser : si des liens amicaux me lient à l'univers de la théologie, rien ne m'y rattache au plan professionnel. C'est pourquoi les lignes qui vont suivre relèvent davantage du senti qu'aucune analyse ne permet vraiment d'étayer. Il appartient donc à mes collègues théologiens de répondre aux questions soulevées en me corrigeant, s'il y a lieu.

Ainsi, à prime abord, la théologie susceptible de m'intéresser me donne souvent l'impression d'être honteuse et d'affirmer ainsi une culpabilité qui la conduit à se soumettre à la problématique des sciences humaines pour se construire comme science religieuse. 
Ainsi que je l'ai déjà exprimé, une telle posture devrait déboucher en toute logique sur le rattachement de la théologie aux sciences humaines où, à terme, elle sera appelée à se consacrer à l'étude des idées aliénantes qui ont jalonné l'histoire de l'humanité. En fait, la théologie ne devient-elle pas simplement un discours scientifique sur les discours de Dieu? Et la question de Dieu la travaille-t-elle encore sous un mode différent de celui des sciences humaines? Si la réponse est positive, cela veut dire que l'interdit de Dieu apparaîtrait au sein même de la théologie. La mort de Dieu s'achèverait donc ainsi par son bannissement d'une théologie soucieuse de faire la preuve de sa scientificité en s'appuyant sur des garants extérieurs qui viennent assurer la mort définitive de Dieu.

Pourtant, le cœur du cœur de la réflexion théologique, n'est-ce pas la question de Dieu? Mais peut-être s'agit-il là aussi de la question "impertinente et désuète " de quelqu'un qui, en raison de son extériorité, n'a pas su prendre la pleine mesure de la scientificité moderne de la théologie. À l'instar du vide social que plusieurs diagnostiquent au sein des sociétés contemporaines, la théologie s'estelle vidée de l'idée de Dieu? Si toutes ces questions s'avèrent fondées, on peut se demander quelle inter-fécondation reste possible, puisqu'alors rien ne différencie fondamentalement la théologie des sciences humaines et que le même interdit les frappe. L'interdisciplinarité ne peut de la sorte que déboucher sur l'enrichissement mutuel de nos ressources culturelles et intellectuelles - ce qui, par les temps qui courent, est à vrai dire déjà beaucoup.

Tandis que les sciences humaines tendent à se vouer à leur application et qu'elles sont en train de jouer un vilain tour à l'humanité en la construisant de manière opérationnelle et, de ce fait, en déshumanisant l'être, la théologie peut-elle nous aider à reconstruire une parole significative en nous permettant de comprendre l'énigme relationnelle que constitue la question de Dieu? Il s'agit peut-être de se laisser aller en quelque sorte à une jonglerie sur l'idée de Dieu qui vient à l'esprit pour lui permettre d'advenir comme humanité. Bien entendu, pour ce faire, la théologie universitaire doit rompre définitivement les amarres avec la théologie dogmatique (ecclésiale?) qui lui fait tellement honte, parce qu'elle nous assomme d'arguments d'autorité fondés sur une vérité révélée à laquelle - modernité oblige - il est évidemment impossible d'acquiescer. 
C'est ainsi que l'on peut s'amuser à formuler une série de questions qui, tout en assurant l'actualité de la réflexion théologique, seraient susceptibles d'alimenter des sciences humaines qui acceptent de se distancier du fantasme de toute-puissance et de reconnaître la finitude de la condition humaine. Ainsi, on pourrait s'interroger sur Dieu comme ce manque dont l'humanité a peut-être besoin pour parvenir à être humaine et renvoyer aux sciences humaines la question de son éradication toujours plus grande qui les rend incapable de contribuer à l'humanisation des sociétés.

On pourrait réfléchir sur cette expérience humaine du Tout Autre agissant comme révélateur. Dieu n'est-il pas, en effet, une Altérité radicale qui permet de fonder l'identité? Ce Tout Autre disparaît de l'horizon des sciences humaines pour se réduire de plus en plus à une altérité fondée sur l'empirisme des différences (le différentialisme) où tend également à s'éroder la commune humanité qui fonde le lien social. Puisque l'expérience religieuse est d'abord et avant tout une expérience symbolique, la théologie peut-elle nous rappeler que poursuivant sur leur lancée, les sciences humaines construisent la désymbolisation de sociétés qui, de ce fait, sont conduites à leur déshumanisation. On pourrait encore prolonger la liste, mais cela ne servira pas à grand-chose si la théologie ne peut renoncer à la réification objectivante de Dieu, à la croyance qui refuse le doute et qui ne peut accepter que Dieu est une question, une idée, un travail humain. Ce faisant, par ailleurs, elle peut, tout en assumant son héritage, sortir de sa soumission "coupable " aux sciences humaines et l'on pourra ainsi en espérer une collaboration qui relève d'une interrogation réciproque.

C'est Alain Renaut qui faisait remarquer que la philosophie doit réexaminer la question du sujet dont la mort ne serait pas si évidente. Ainsi notait-il que :

... le problème du sujet nous est en grande partie revenu, à nous, philosophes, à partir des sciences sociales et des interrogations qu'elles ont été conduites à formuler sur elles-mêmes. Le fait, en lui-même, ne m'étonne pas, à une époque où les philosophes semblent se vouer à consacrer leurs principaux efforts à dévoiler 
les derniers secrets de la théorie malebranchiste des tourbillons ou à broder de subtiles variations sur la forme d'un tympan ${ }^{18}$.

N'en irait-il pas de même pour la question de Dieu qui mériterait d'être réexaminée par une théologie des temps présents? À l'heure où domine la pensée utilitaire et productiviste qui réclame que la recherche soit pertinente, de telles questions non pertinentes et impertinentes sont peut-être la voie d'un renouveau de la pensée. Le chantier est certes immense, il m'apparaît à la mesure de notre grandeur ... divine!

18 A. RENAUT, «Les subjectivités : pour une histoire du concept de sujet», dans E. GUIBERT-SLEDZIEWSKI et J.-L. VIEILLARD-BARON (dir.), Penser le sujet aujourd'hui, Paris. Méridiens Klincksieck, 1988, p. 74. 